\title{
Prevalência de pré-diabetes e hiperglicemia intermediária em adultos e fatores associados, Pesquisa Nacional de Saúde
}

\author{
Prediabetes and intermediate hyperglycemia prevalence \\ in adults and associated factors, Health National Survey
}

Betine Pinto Moehlecke Iser (https://orcid.org/0000-0001-6061-2541) ${ }^{1}$

Pedro Cisalpino Pinheiro (https://orcid.org/0000-0002-6954-1708) ${ }^{2}$

Deborah Carvalho Malta (https://orcid.org/0000-0002-8214-5734) ${ }^{2}$

Bruce Bartholow Duncan (http://orcid.org/0000-0002-7491-2630) ${ }^{3}$

Maria Inês Schmidt (http://orcid.org/0000-0002-3837-0731) ${ }^{3}$

\footnotetext{
${ }^{1}$ Programa de PósGraduação em Ciências da Saúde, Universidade do Sul de Santa Catarina. Av. José Acácio Moreira 787, Dehon. 88704-900 Tubarão SC Brasil.betinee@gmail.com ${ }^{2}$ Escola de Enfermagem, Universidade Federal de Minas Gerais. Belo Horizonte MG Brasil. ${ }^{3}$ Programa de PósGraduação em Epidemiologia, Universidade Federal do Rio Grande do Sul. Porto Alegre RS Brasil.
}

\begin{abstract}
This study aimed to evaluate the prevalence of prediabetes and intermediate hyperglycemia in Brazilian adults, according to different diagnostic criteria, and establish associated factors to its occurrence. We analyzed the National Health Survey laboratory data collected from 2014 to 2015. The prevalence of the conditions was calculated according to the American Diabetes Association (ADA) diagnostic criteria based on glycated hemoglobin (HbAlc) 5.7\%-6.4\%, and the World Health Organization (WHO) 6-6.4\%, among those without criteria for diabetes. Crude and adjusted prevalence rates (PR) and 95\% CI were calculated using Poisson regression with robust variance. The prevalence of prediabetes by ADA and WHO criteria was 18.5 and 7.5\%, respectively. We observed a gradient of increased prevalence by the age of the population and risk factors, like arterial hypertension, obesity, elevated waist circumference, and low HDL cholesterol levels. Less educated people and the self-declared black had a higher prevalence. This study pointed out a range from 7.5 to $18.5 \%$ of Brazilian adults with prediabetes and intermediate hyperglycemia and identified a risk score to this condition's occurrence.
\end{abstract}

Key words Hyperglycemia, Prediabetic state, Glycated Hemoglobin A, Epidemiological surveys, Risk factors
Resumo O objetivo deste estudo foi avaliar a prevalência de pré-diabetes e hiperglicemia intermediária em adultos brasileiros, considerando diferentes critérios diagnósticos, e estabelecer fatores associados à sua ocorrência. Análise dos dados laboratoriais da Pesquisa Nacional de Saúde, coletados em 2014 e 2015. Foram calculadas as prevalências das condições conforme critérios da Associação Americana de Diabetes (ADA) Hemoglobina Glicada (HbAlc) 5,7 a 6,4\% -e da Organização Mundial de Saúde (OMS), de $6-6,4 \%$ entre aqueles que não tinham critério para diabetes. Razões de prevalência (RP) brutas e ajustadas e IC $95 \%$ foram calculados por regressão de Poisson com variância robusta. A prevalência de pré-diabetes pelo critério ADA foi de 18,5\% e de 7,5\% pelo critério da OMS. Verificou-se um gradiente de aumento das prevalências segundo a idade da população e presença de fatores de risco como hipertensão arterial, obesidade, circunferência abdominal elevada e baixo colesterol HDL. Os menos escolarizados e os declarados pretos apresentaram prevalências superiores. Este estudo aponta um intervalo entre 7,5 a 18,5\% de adultos brasileiros que apresentam pré-diabetes e hiperglicemia intermediária, além de identificar um escore de risco para a ocorrência dessa condição.

Palavras-chave Hiperglicemia, Estado pré-diabético, Hemoglobina A Glicada, Inquéritos epidemiológicos, Fatores de risco 


\section{Introdução}

Estados metabólicos intermediários caracterizados por níveis glicêmicos elevados, acima do considerado "normal", apresentam um risco aumentado para diabetes mellitus e suas complicações, incluindo as doenças cardiovasculares ${ }^{1}$. Estimase que, anualmente, 5 a $10 \%$ das pessoas com a condição intermediária progridam para diabetes mellitus tipo $2^{2}$. Revisão sistemática de Zhang ${ }^{3}$ indicou que valores de HbAlc entre 6-6,5\% estão associados a uma incidência de 25 a 50\% de DM em cinco anos.

Por outro lado, por ser considerada uma condição intermediária, esta é passível de estabilização frente à adoção de um estilo de vida saudável, como controle alimentar, redução/manutenção do peso e atividade física regular. Assim, sua detecção precoce pode servir de alerta para a identificação de indivíduos de alto risco, elegíveis para estratégias preventivas mais rígidas, criando-se oportunidades de controle adequado.

A Associação Americana de Diabetes (ADA) denomina esta condição de 'pré-diabetes' e recomenda seu rastreamento em indivíduos assintomáticos quando houver fatores de risco associados, tais como obesidade, hipertensão arterial e dislipidemias'. A Organização Mundial de Saúde contraindica o uso do termo pré-diabetes, preferindo chamar de 'hiperglicemia intermediária"4.

Embora os critérios diagnósticos tenham sido muito debatidos nos últimos anos, ainda não há consenso sobre os limites a serem considerados para este estágio intermediário, em termos de aumento de complicações, considerando os pontos de corte estabelecidos para diabetes, de $7 \mathrm{mmol} / \mathrm{L}$ ou $126 \mathrm{mg} / \mathrm{dL}$ de glicemia de jejum, ou $6,5 \%$ de hemoglobina glicada ( $\mathrm{HbAlc}$ ), conforme demonstrado no Quadro $1^{1,4,5}$.
Considerando as divergências de critérios e nomenclaturas, as estimativas de prevalência de pré-diabetes/ hiperglicemia intermediária são escassas. A Federação Internacional de Diabetes (do Inglês, International Diabetes Federation - IDF), considerando os níveis de intolerância à glicose propostos pela Organização Mundial de Saúde (OMS) - glicemia de jejum $<126 \mathrm{mg} / \mathrm{dL}$ e teste de tolerância oral à glicose (TOTG) $\geq 140 \mathrm{e}<200$ $\mathrm{mg} / \mathrm{dL}$ - aponta um contingente maior do que 370 milhões de pessoas no mundo com essa condição, representando uma prevalência de 7,5\%. Mais de $70 \%$ destes pertencem a países de baixamédia renda e cerca de $28 \%$ estão na faixa etária de 20 a 39 anos de idade. Para a América Central e do Sul, a prevalência estimada é de $9,7 \%{ }^{6}$.

A Sociedade Brasileira de Diabetes (SBD) adota critérios semelhantes à $\mathrm{ADA}$, sendo considerado pré-diabetes quando glicemia de jejum entre 100 e 126 mg/dL (glicemia de jejum alterada) ou tolerância à glicose diminuída com valores $2 \mathrm{~h}$ após teste oral de tolerância à glicose (TOTG) entre 140-199 mg/dL (intolerância oral à glicose), ou valores de hemoglobina glicada (HbAlc) entre 5,7 e 6,4\% ${ }^{7}$.

A medida de $\mathrm{HbAlc}$ é aceita para rastreamento de pré-diabetes e diabetes pela $\mathrm{ADA} / \mathrm{SBD}$ e, além de ser considerada mais conveniente por não precisar de jejum, reflete um valor mais estável, referente a um período anterior (entre 8 a 12 semanas), e não dependente de variabilidades momentâneas. No entanto, é influenciada por fatores como etnia, idade e hemoglobinopatias, tendo algumas limitações ao seu uso de forma isolada ${ }^{8}$.

A adoção de critérios diagnósticos distintos pode ser influenciada por políticas específicas e terão impacto na qualidade de vida e nas despesas em saúde da população, podendo onerar so-

Quadro 1. Critérios laboratoriais e pontos de corte para diagnóstico de pré-diabetes/ hiperglicemia intermediária e diabetes, segundo entidade proponente.

\begin{tabular}{|l|c|c|c|}
\hline \multicolumn{1}{|c|}{ Critérios / Pontos de corte } & ADA / SBD & OMS / IDF / IEC & Todos \\
\hline Glicemia de jejum & Pré-diabetes & $\begin{array}{c}\text { Hiperglicemia } \\
\text { Intermediária }\end{array}$ & Diabetes \\
\hline Teste oral de tolerância à glicose & $\begin{array}{c}100-125 \mathrm{mg} / \mathrm{dL}^{*} \\
(5,6-6,9 \mathrm{mmol} / \mathrm{L})\end{array}$ & $\begin{array}{c}110-125 \mathrm{mg} / \mathrm{dL} \\
(6,1-6,9 \mathrm{mmol} / \mathrm{L})\end{array}$ & $\begin{array}{c}126 \mathrm{mg} / \mathrm{dL}(<7,0 \\
\mathrm{mmol} / \mathrm{L})\end{array}$ \\
\hline TOTG) 2h após sobrecarga 75g glicose & $\begin{array}{c}140-199 \mathrm{mg} / \mathrm{dL} \\
(7,8-11,0 \mathrm{mmol} / \mathrm{L})^{\#}\end{array}$ & $\begin{array}{c}140 \mathrm{e} 199 \mathrm{mg} / \mathrm{dl} \\
(7,8-11,0 \mathrm{mmol} / \mathrm{L})^{\#}\end{array}$ & $\begin{array}{c}\geq 200 \mathrm{mg} / \mathrm{dL} \\
(11,1 \mathrm{mmol} / \mathrm{L})\end{array}$ \\
\hline Hemoglobina glicada (HbAlc) & $\begin{array}{c}5,7-6,4 \% \\
(39-47 \mathrm{mmol} / \mathrm{mol})\end{array}$ & $\begin{array}{c}26-6,4 \% \\
(42-47 \mathrm{mmol} / \mathrm{mol})\end{array}$ & \begin{tabular}{c}
$\geq 8 \mathrm{mmol} / \mathrm{mol})$ \\
\hline
\end{tabular}
\end{tabular}

ADA: American Diabetes Association; SBD: Sociedade Brasileira de Diabetes; OMS: Organização Mundial de Saúde; IDF: International Diabetes Federation; IEC: International Expert Committee.

*Categoria conhecida como glicemia de jejum alterada. ${ }^{*}$ categoria conhecida como intolerância à glicose diminuída. 
bremaneira os serviços públicos. Por outro lado, um critério menos sensível pode perder a oportunidade de diagnosticar e tratar precocemente as complicações relacionadas à doença.

O objetivo deste estudo foi avaliar a prevalência de hiperglicemia intermediária e pré-diabetes em adultos brasileiros, considerando diferentes critérios diagnósticos, e estabelecer fatores associados à sua ocorrência.

\section{Métodos}

Foram analisados os dados laboratoriais da Pesquisa Nacional de Saúde (PNS), entre os anos de 2014 e 2015. A PNS é uma pesquisa de base domiciliar, de âmbito nacional, que utiliza amostras probabilísticas em três estágios. As unidades primárias de amostragem foram os setores censitários ou conjunto de setores, as unidades secundárias, os domicílios e as unidades terciárias, os residentes adultos, com idade igual ou maior que 18 anos.

As entrevistas foram realizadas em 64.348 domicílios e 60.202 moradores responderam ao questionário individual. Inicialmente foram selecionados 25\% dos setores censitários para a realização dos exames laboratoriais e, supondose uma taxa de não resposta de $20 \%$, o número esperado de indivíduos com dados laboratoriais era de, aproximadamente, $12 \mathrm{mil}^{9}$. A subamostra foi selecionada com probabilidade proporcional ao inverso da distância do município onde se localiza a unidade primária de amostragem e o município mais próximo com 80 mil habitantes ou mais (relativa à dificuldade de coleta), em todas as Unidades da Federação. Considerando as perdas na subamostra de indivíduos indicados para a realização dos exames laboratoriais, pela dificuldade de localização do endereço pelo laboratório contratado e/ou a recusa do morador selecionado em realizar a coleta de material biológico, foram coletados exames em 8.952 pessoas. Para correções de possíveis vieses foram utilizadas ponderações do processo amostral e realizados pesos de pós-estratificação, segundo sexo, idade, escolaridade e região.

A HbA1c foi colhida em tubo com ácido etilenodiamino tetra-acético (EDTA) e dosada por cromatografia líquida de alta performance por troca iônica (high pressure liquid chromatography - HPLC), em laboratório certificado pelo $\mathrm{Na}$ tional Glicohemoglobin Standardization Program. A coleta de sangue periférico foi realizada a qualquer hora do dia, sem jejum ${ }^{10}$.
Para as análises, considerou-se inicialmente a comparação de diferentes critérios de diagnóstico: o ponto de corte estabelecido pela ADA para pré-diabetes $5,7 \% \geq \mathrm{HbA} 1 \mathrm{c} \leq 6,4 \%{ }^{1}$ e um intervalo mais estreito aceito pela OMS para o estado de hiperglicemia intermediária: $6 \% \geq \mathrm{HbA} 1 \mathrm{c} \leq$ $6,4 \%{ }^{4}$. Foi calculada a prevalência de pré-diabetes e hiperglicemia intermediária entre os entrevistados, excluindo-se aqueles com glicemia $\geq$ $6,5 \%$, ou seja, que atendiam critério para diabetes, independente de já ter o diagnóstico da doença. Também foram excluídos indivíduos com informações faltantes para as variáveis de interesse do estudo, descritas a seguir. As prevalências foram calculadas segundo características sociodemográficas: sexo, idade, escolaridade, raça/cor, região do país; índice de massa corporal (IMC), sendo categorizado em eutrofia e baixo peso (< $25 \mathrm{~kg} / \mathrm{m} 2$ ), sobrepeso (entre 25 e $29,9 \mathrm{~kg} / \mathrm{m} 2$ ) e obesidade ( $\geq 30 \mathrm{~kg} / \mathrm{m} 2$ ), e circunferência abdominal, obtida no ponto médio entre a borda inferior da última costela e a borda superior da crista ilíaca, a qual pode ser considerada um indicador da gordura visceral. A medida igual ou superior a $102 \mathrm{~cm}$ em homens e $88 \mathrm{~cm}$ em mulheres indica risco cardiovascular e por isso foi considerada neste estudo. Foi ainda verificada a presença de outros fatores de risco, como hipertensão arterial (definida pela pressão arterial $\geq 140$ e/ou 90 $\mathrm{mmHg}$, pelo relato de diagnóstico médico de hipertensão arterial ou de uso de medicamento anti-hipertensivo), e baixo nível de colesterol HDL (do inglês, High Density Lipoproteins), sendo considerado como ponto de corte, para homens $<40 \mathrm{mg} / \mathrm{dl}$ e para mulheres $<50 \mathrm{mg} / \mathrm{dl}$, medido na mesma amostra de sangue. Foram considerados ainda dois fatores de risco comportamentais: a inatividade física e o baixo consumo de frutas e legumes. Foi considerado inativo quem não realizou qualquer atividade física no lazer nos últimos três meses, e insuficiente ativo quem não alcançou, pelo menos, 150 minutos semanais de atividade física considerando o lazer, o trabalho e o deslocamento. O baixo consumo de frutas e verduras foi identificado naqueles que não relataram ingestão desses alimentos em cinco vezes ao dia em, pelo menos, cinco dias na semana. Ainda, foi calculado o quanto a ocorrência simultânea destes fatores reconhecidamente de risco afeta a prevalência de pré-diabetes/hiperglicemia intermediária.

As análises foram realizadas com o auxílio do programa estatístico Data Analysis and Statistical Software (Stata), versão 14, usando-se o comando survey para incorporação dos pesos de pós-estra- 
tificação. Realizaram-se análise bivariada e cálculo das prevalências, com intervalo de confiança de $95 \%$ (IC95\%). Foram calculadas as razões de prevalência (RP) pelo método de regressão de Poisson com variância robusta, brutas e ajustadas por análise multivariada, considerando as demais variáveis de interesse. Para avaliação por escore de risco, considerou-se o número de fatores de risco presentes no mesmo indivíduo, entre: circunferência abdominal aumentada, obesidade, hipertensão, colesterol elevado, inatividade física e baixo consumo de frutas e verduras, além da idade ( $\geq 60$ anos).

A PNS foi aprovada pela Comissão Nacional de Ética em Pesquisa (CONEP). Os participantes da pesquisa assinaram o Termo de Consentimento Livre e Esclarecido (TCLE) e autorizaram a coleta de exames laboratoriais.

\section{Resultados}

De um total de 8.541 indivíduos com análise da $\mathrm{HbA1c}$, foram considerados neste estudo 7.548 participantes na amostra, pois foram excluídos 595 entrevistados com nível de HbAlc compatível com diabetes $(\geq 6,5 \%$, sendo 310 com relato de diagnóstico prévio) e outros 398 por informações faltantes.

A prevalência de pré-diabetes, considerando critérios da ADA, foi de 18,5\% (IC 95\% 17,419,7), sem diferença significativa segundo sexo. Pelos critérios recomendados pela OMS, a prevalência de hiperglicemia intermediária foi mais de duas vezes menor, sendo de 7,5\% (IC 95\% 6,78,3), conforme detalhado na Tabela 1.

Em ambos os critérios, verifica-se um gradiente de aumento das prevalências segundo a idade, chegando a ser quatro vezes maior na população idosa ( $\geq 60$ anos). Por outro lado, os mais escolarizados apresentaram menor prevalência de pré-diabetes / hiperglicemia intermediária, quando comparados àqueles com até oito anos de estudo.

Em relação à raça/cor, diferenças significativas foram observadas, em análise ajustada, com maior prevalência para os declarados pretos em ambos os critérios. Uma menor prevalência para os declarados pardos foi verificada apenas em análise bruta, pelo critério da ADA. Entre as macrorregiões do Brasil, o Sudeste apresentou prevalência superior, em ambos os critérios, e o Centro-Oeste, pelo critério da ADA.

Entre as morbidades investigadas, o excesso de peso, pelo critério da ADA, e a obesidade, por ambos os critérios, foram fatores relacionados à maior prevalência de pré-diabetes / hiperglicemia intermediária. $\mathrm{O}$ aumento da circunferência abdominal, a presença de hipertensão arterial e o baixo colesterol HDL foram associados à maior prevalência de pré-diabetes/hiperglicemia intermediária.

A inatividade física foi relacionada à maior prevalência de pré-diabetes/hiperglicemia intermediária em comparação aos considerados ativos apenas em análises brutas. Embora pelo critério da OMS a prevalência de hiperglicemia intermediária tenha pontualmente reduzido nos adultos considerados ativos fisicamente, em análise ajustada as diferenças não foram estatisticamente significativas. Da mesma forma, o consumo de frutas e verduras não apresentou relação com as prevalências verificadas.

$\mathrm{Na}$ Tabela 2, é apresentado o escore de risco, considerando apenas a população com pelo menos um fator $(n=7.297)$. Verifica-se que, a partir de três fatores de risco incorporados, a prevalência do estado de pré-diabetes/hiperglicemia intermediária aumenta, independentemente do critério diagnóstico utilizado, embora com o ponto de corte da OMS essas diferenças sejam mais pronunciadas. A presença de cinco ou mais fatores de risco aumenta em maior proporção a prevalência, com razões de prevalência até quatro vezes superiores do que aqueles que apresentam um fator de risco.

A Figura 1 apresenta as prevalências brutas segundo critério diagnóstico e variáveis independentes. É possível verificar a maior prevalência a partir do ponto de corte da ADA, ao mesmo tempo que, para algumas variáveis, como raça/ cor e inatividade física, maiores diferenças entre os estratos aparecem com o critério adotado pela OMS: entre pretos e outros, incluindo amarelo e indígena, e entre os inativos e os ativos.

\section{Discussão}

Neste estudo verificou-se a prevalência e os fatores associados ao que se convenciona denominar, pela ADA, de pré-diabetes, e pela OMS, de hiperglicemia intermediária. Este é considerado um estágio intermediário, prévio ao estabelecimento do diabetes, mas que já poderia estar relacionado ao risco de complicações decorrentes da doença. $\mathrm{O}$ estudo apontou um intervalo entre $7,5 \mathrm{a}$ $17,5 \%$ de adultos brasileiros que apresentam prédiabetes e hiperglicemia intermediária, segundo diferentes critérios diagnósticos. Verificou-se um 
Tabela 1. Prevalências e Razão de prevalências de hiperglicemia intermediária e pré-diabetes por variáveis sociodemográficas e fatores de risco, baseados em critérios ADA e OMS, PNS 2014-2015 ( $\mathrm{N}=7.548)$.

\begin{tabular}{|c|c|c|c|c|c|c|}
\hline \multirow[b]{2}{*}{ Variáveis } & \multicolumn{3}{|c|}{ ADA: $5,7-6,4 \%$} & \multicolumn{3}{|c|}{ OMS: $6-6,4 \%$} \\
\hline & $\begin{array}{c}\text { Prev. \% (IC } \\
95 \%)\end{array}$ & RPbruta & $\mathbf{R P a j}^{\infty}$ & Prev. (IC 95\%) & RPbruta & $\mathbf{R P a j}^{\infty}$ \\
\hline Total & $18,5(17,4--19,7)$ & - & - & $7,5(6,7-8,3)$ & - & - \\
\hline \multicolumn{7}{|l|}{ Sexo } \\
\hline Masculino & $17,2(15,6-19)$ & 1 & 1 & $7,0(5,9-8,3)$ & 1 & 1 \\
\hline Feminino & $19,7(18,2-21,2)$ & $1,14^{*}$ & 0,99 & $7,9(6,9-9)$ & 1,12 & 0,9 \\
\hline \multicolumn{7}{|l|}{ Faixa etária } \\
\hline $18-39$ & $7,8(6,6-9,2)$ & 1 & 1 & $2,2(1,6-3,1)$ & 1 & 1 \\
\hline $40-59$ & $20,4(18,5-22,4)$ & $2,62^{\star}$ & $2,15^{\star}$ & $7,8(6,6-9,3)$ & $3,5^{\star}$ & $2,53^{\star}$ \\
\hline$\geq 60$ anos & $37,6(34,7-40,5)$ & $4,82^{\star}$ & $3,63^{\star}$ & $17,9(15,6-20,4)$ & $7,98^{\star}$ & $4,83^{\star}$ \\
\hline \multicolumn{7}{|l|}{ Escolaridade } \\
\hline $\begin{array}{l}\text { Sem instrução até } \\
\text { fundamental }\end{array}$ & $22(20,4-23,6)$ & 1 & 1 & $9,8(8,6-11)$ & 1 & 1 \\
\hline Médio completo ou mais & $14,5(13-16,3)$ & $0,66^{*}$ & 0,95 & $4,9(4-5,9)$ & $0,5^{\star}$ & 0,79 \\
\hline \multicolumn{7}{|l|}{ Raça/cor } \\
\hline Branca & $18,8(17,1-20,6)$ & 1 & 1 & $7,3(6,3-8,6)$ & 1 & 1 \\
\hline Preta & $26,7(22,3-31,6)$ & $1,42^{\star}$ & $1,5^{\star}$ & $13,8(10,3-18,4)$ & $1,89^{*}$ & $2,07^{\star}$ \\
\hline Parda & $16,2(14,8-17,7)$ & $0,86^{*}$ & 0,97 & $6,2(5,4-7,3)$ & 0,85 & 1,04 \\
\hline Outra & $25,9(14,7-41,5)$ & 1,38 & 1,51 & $8,6(2,6-25,3)$ & 1,18 & 1,36 \\
\hline \multicolumn{7}{|l|}{ Região } \\
\hline Norte & $14,6(13-16,3)$ & 1 & 1 & $5,3(4,4-6,5)$ & 1 & 1 \\
\hline Nordeste & $16,4(15-17,9)$ & 1,13 & 1,02 & $5,8(4,9-6,7)$ & 1,08 & 0,95 \\
\hline Sudeste & $20,6(18,5-22,9)$ & $1,42^{\star}$ & $1,19^{*}$ & $9,1(7,7-10,8)$ & $1,71^{\star}$ & $1,4^{\star}$ \\
\hline Sul & $16,7(14,4-19,3)$ & 1,15 & 0,97 & $6,8(5,4-8,6)$ & 1,28 & 1,07 \\
\hline Centro-Oeste & $20,2(17,5-23,2)$ & $1,39^{*}$ & $1,26^{\star}$ & $7,0(5,4-9,1)$ & 1,31 & 1,18 \\
\hline \multicolumn{7}{|l|}{ IMC } \\
\hline Baixo peso/normal & $12,4(11-14)$ & 1 & 1 & $4,8(3,8-5,9)$ & 1 & 1 \\
\hline Excesso de peso & $19,8(17,9-21,8)$ & $1,59^{*}$ & $1,27^{\star}$ & $7,9(6,7-9,3)$ & $1,65^{\star}$ & 1,19 \\
\hline Obesidade & $29,3(26,4-32,4)$ & $2,35^{\star}$ & $1,65^{\star}$ & $12,7(10,7-15)$ & $2,67^{\star}$ & $1,54^{*}$ \\
\hline \multicolumn{7}{|l|}{ Circunferência abdominal } \\
\hline Normal & $13(11,8-14,4)$ & 1 & 1 & $4,5(3,8-5,4)$ & 1 & 1 \\
\hline Aumentada & $27,7(25,7-29,8)$ & $2,13^{*}$ & $1,2^{*}$ & $12,4(11-14,1)$ & $2,73^{\star}$ & $1,51^{\star}$ \\
\hline Hipertensão & & & & & 0 & \\
\hline Não & $12,6(11,5-13,9)$ & 1 & 1 & $4,2(3,5-5)$ & 1 & 1 \\
\hline Sim & $30(27,8-32,4)$ & $2,38^{*}$ & $1,32^{\star}$ & $14,0(12,3-15,9)$ & $3,35^{\star}$ & $1,55^{\star}$ \\
\hline \multicolumn{7}{|l|}{ Colesterol HDL } \\
\hline Recomendado & $15,2(13,8-16,8)$ & 1 & 1 & $5,9(5-6,9)$ & 1 & 1 \\
\hline Baixo & $22(20,3-23,8)$ & $1,45^{\star}$ & $1,28^{\star}$ & $9,2(8,1-10,5)$ & $1,57^{\star}$ & $1,35^{\star}$ \\
\hline Inatividade física & & & & & 0 & \\
\hline Inativo & $20,2(18,8-21,6)$ & 1 & 1 & $8,3(7,4-9,4)$ & 1 & 1 \\
\hline Insuficiente ativo & $14,6(11,4-18,5)$ & $0,72^{\star}$ & 0,87 & $7,6(5,3-10,7)$ & 0,91 & 1,16 \\
\hline Ativo & $15(12,9-17,4)$ & $0,75^{\star}$ & 0,96 & $5,0(3,9-6,6)$ & $0,61^{\star}$ & 0,85 \\
\hline \multicolumn{7}{|l|}{ Consumo de frutas e verduras } \\
\hline Adequado/Regular & $18,4(16,5-20,5)$ & 1 & 1 & $7,6(6,4-9,2)$ & 1 & 1 \\
\hline Baixo & $18,5(17,2-20)$ & 1,01 & 1,11 & $7,4(6,5-8,4)$ & 0,97 & 1,05 \\
\hline
\end{tabular}

Fonte: PNS 2014-2015.

Prev. $=$ Prevalência. $\mathrm{RP}=$ Razão de Prevalência. ${ }^{\infty} \mathrm{RPaj}=\mathrm{RP}$ ajustada por análise multivariada considerando todas as variáveis do modelo. ${ }^{*}$ Prevalências com diferenças significativas, considerando nível de significância de $5 \%$. 
Tabela 2. Prevalência e Razão de prevalências de hiperglicemia intermediária pelo número de fatores de risco**, baseados em critérios ADA e OMS, PNS 2014-2015 ( $\mathrm{n}=7.297)$.

\begin{tabular}{lrrrrrrr}
\hline \multirow{2}{*}{ Variáveis } & \multicolumn{3}{c}{ ADA } & \multicolumn{3}{c}{ OMS } \\
\cline { 2 - 7 } & Prev. \% (IC 95\%) & RPbruta & RPaj $^{\infty}$ & Prev. \% (IC 95\%) & RPbruta & RPaj $^{\infty}$ \\
\hline Um fator & $9,1(7,0-11,8)$ & 1 & 1 & $2,4(1,4-4,1)$ & 1 & 1 \\
2 fatores & $12,0(10,2-14,0)$ & 1,31 & 1,13 & $4,3(3,3-5,7)$ & 1,82 & 1,49 \\
3 a 4 & $22,3(20,5-24,2)$ & $2,44^{*}$ & $1,76^{*}$ & $9,1(7,9-10,5)$ & $3,82^{*}$ & $2,49^{*}$ \\
5 ou mais & $35,9(32,1-39,9)$ & $3,93^{*}$ & $2,56^{*}$ & $17,2(14,3-20,6)$ & $7,23^{*}$ & $4,13^{*}$ \\
\hline
\end{tabular}

Fonte: PNS 2014-2015.

Prev. $=$ Prevalência. $\mathrm{RP}=$ Razão de Prevalência. ${ }^{\infty} \mathrm{RPaj}=\mathrm{RP}$ ajustada por análise multivariada considerando todas as variáveis relacionadas no escore $\left(1,2,3\right.$ ou 4,5 ou mais) e a idade como variável de controle. ${ }^{\star}$ Prevalências com diferenças significativas, considerando nível de significância de 5\%. ${ }^{\star *}$ Considerando as variáveis: obesidade, circunferência abdominal elevados, hipertensão arterial, baixo colesterol HDL, inatividade física e baixo consumo de frutas e verduras.

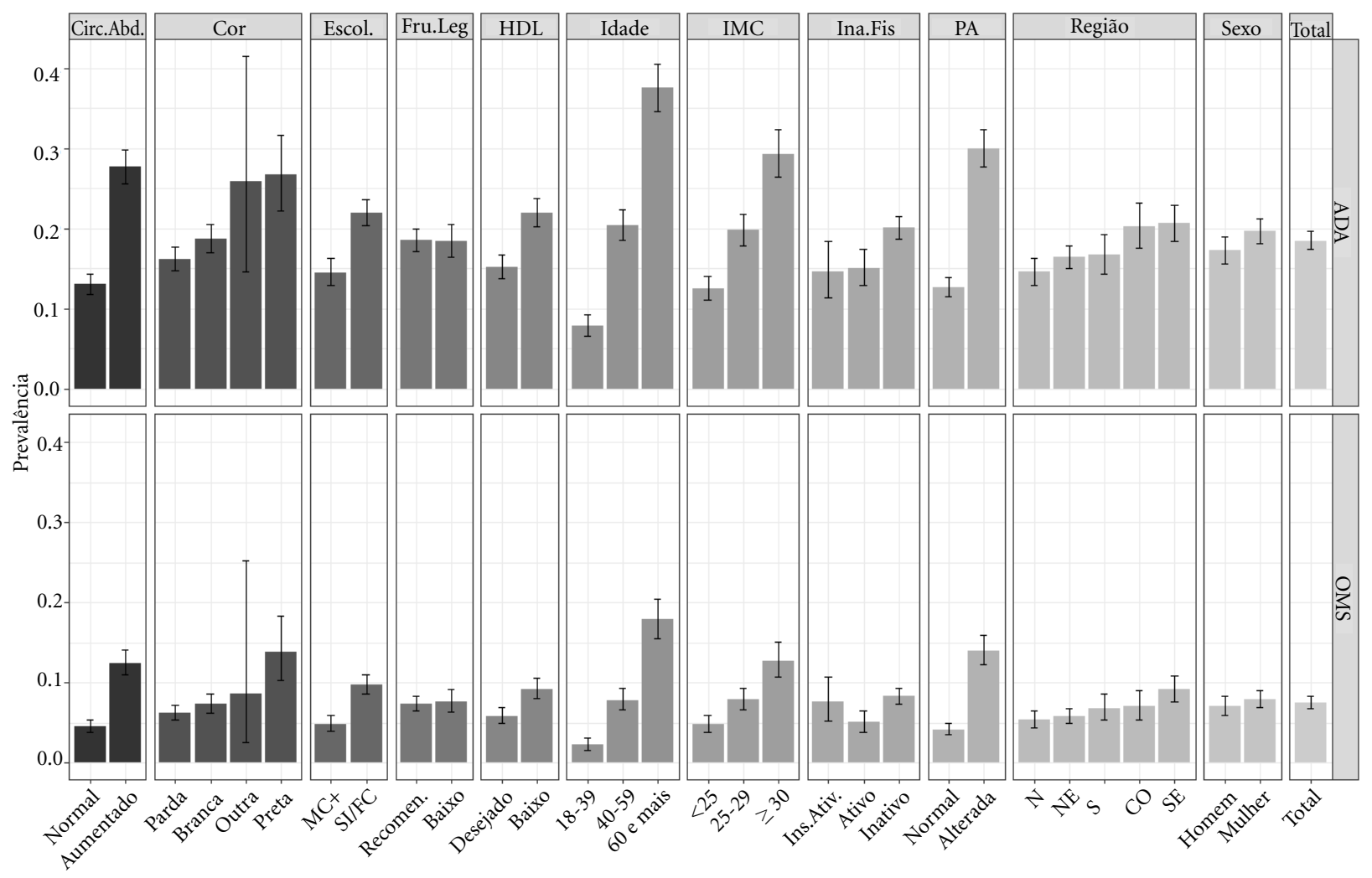

Figura 1. Prevalência de pré diabetes e hiperglicemia intermediária de acordo com características sociodemográficas e fatores de risco, baseados em critérios ADA e OMS, PNS 2014-2015.

Fonte: PNS 2014-2015.

Siglas: Circ.Abd = circunferência abdominal Aumentada: $\geq 102 \mathrm{~cm}$ em homens $/ 88 \mathrm{~cm}$ em mulheres. HDL (do inglês, High Density Lipoproteins); Baixo: $<40 \mathrm{mg} / \mathrm{dl}$ para homens e $<50 \mathrm{mg} / \mathrm{dl}$ para mulheres. Escol. = escolaridade. $\mathrm{MC}+=$ médio completo ou mais SI/FC = sem instrução até fundamental completo. Fru.Leg. $=$ consumo de frutas e verduras; Recomen. $=$ recomendado $(\geq 5$ porções ao dia em cinco ou mais vezes na semana $)$. IMC $=$ Índice de Massa Corporal. Ina.Fis. $=$ Inatividade física Ins.Ativ. $=$ insuficientemente ativo $(<150$ minutos/semana $)$. PA $=$ Pressão Arterial PA Alter. $=$ pressão arterial alterada ou elevada ( $\geq 140$ e/ou $90 \mathrm{mmHg}$, relato de diagnóstico médico ou uso de medicamento anti-hipertensivo). $\mathrm{N}=$ Região Norte; $\mathrm{NE}=\mathrm{Nordeste;} \mathrm{S}=$ Sul; $\mathrm{CO}=$ Centro-Oeste; $\mathrm{SE}=$ Sudeste. $\mathrm{ADA}=$ Associação Americana de Diabetes. OMS = Organização Mundial da Saúde. 
gradiente de aumento das prevalências segundo a idade da população e presença de fatores de risco como hipertensão arterial, obesidade, circunferência abdominal elevada e baixo HDL. Os menos escolarizados e os declarados pretos apresentaram prevalências superiores. Dessa forma, pode-se identificar um conjunto de fatores de risco comuns à ocorrência do pré-diabetes e da hiperglicemia intermediária.

A prevalência variou de acordo com o ponto de corte estabelecido para os valores de hemoglobina glicada. De toda forma, pode-se considerar que, pelo menos, 7,5\% da população adulta brasileira tenha níveis glicêmicos acima da normalidade, mas abaixo do ponto de corte para diabetes, enfatizando-se a necessidade de medidas preventivas quanto aos fatores de risco da doença.

Embora a prevalência de DM autorreferido em diferentes inquéritos ${ }^{11-14}$ e aferida pela própria PNS, quando considerado $\mathrm{HbA} 1 \mathrm{c} \geq 6,5 \%{ }^{10}$, seja maior entre as mulheres, no presente estudo não foram verificadas diferenças na frequência de pré-diabetes/hiperglicemia intermediária segundo sexo, estando de acordo com os resultados do IDF $^{6}$.

Por outro lado, o padrão verificado, de aumento da prevalência segundo a idade da população, com um gradiente de risco dado pela baixa escolaridade, a obesidade e a presença de comorbidades como hipertensão arterial, além da inatividade física, está de acordo com a ocorrência do diabetes em $\mathrm{si}^{10-12}$. Porém, neste estudo, pode-se observar que a determinação do critério diagnóstico influencia nessas relações.

O critério da OMS pode ser considerado mais restritivo, no sentido de que a prevalência é reduzida a mais da metade em comparação aos critérios adotados pela ADA; pode-se deduzir, desta forma, que há uma parcela importante da população no intervalo de glicemia limítrofe entre os dois critérios (HbAlc entre 5,8-5,9), sendo fundamental incluí-las nos esforços preventivos no sentido de postergar o estabelecimento da doença. A baixa concordância entre os testes, com o dobro da estimativa de prevalência com os critérios da ADA, já tinha sido identificada em revisão sistemática de Barry et al. ${ }^{15}$ A prevalência encontrada ao utilizar os critérios indicados pelo Comitê Internacional de Especialistas (IEC) da OMS foi de 27\% de prédiabetes, sendo $48 \%$ destes identificados apenas pelo aumento da $\mathrm{HbA1c}$, enquanto considerando critérios da ADA, a prevalência seria de 49\%, com maior sobreposição de resultados.

A comparação das estimativas verificadas neste estudo com a literatura fica limitada pela diversidade de critérios utilizados e por uma variedade de fatores que interferem na prevalência desta condição, incluindo as características específicas da população ${ }^{5}$. Em revisão sistemática, a prevalência de pré-diabetes apresentou ampla variação, de $27 \%$ a $66 \%$ e, considerando apenas valores anormais de $\mathrm{HbAlc}$, variou de 8 a $48 \%$ em diferentes populações ${ }^{15}$.

São poucos os estudos brasileiros de base populacional que trazem estimativas de pré-diabetes e hiperglicemia intermediária. Estudo realizado na década de 1980 em nove cidades brasileiras ${ }^{16}$ verificou uma prevalência de intolerância à glicose de $7,8 \%$ na população de $30-69$ anos. No interior do Ceará, em amostra aleatória da população $\geq 20$ anos, a prevalência de pré-diabetes foi de 14,2\% (IC95\% 11,6-16,7), sendo esta determinada por: glicemia de jejum entre 110 e $126 \mathrm{mg} /$ $\mathrm{dL}$ e por um resultado do TOTG $<140 \mathrm{mg} / \mathrm{dL}^{17}$. Tendo como referência o critério da OMS, o uso da HbAlc apresentou área sob a curva ROC (do inglês, Receiver Operating Characteristic) para detecção de pré-diabetes de $61 \%$, sendo indicado um ponto de corte ideal de $6,0 \%$ (42 $\mathrm{mmol} / \mathrm{mol})$, com sensibilidade de $67,3 \%$ e especificidade de $52 \%{ }^{17}$. Uma avaliação de 138 pacientes considerados de alto risco para o desenvolvimento de diabetes, pela presença de condições metabólicas, como hipertensão, obesidade e dislipidemia, foi realizada em um hospital público de São Paulo ${ }^{18}$. A prevalência de estado pré-diabético foi $68,0 \%$, sendo que aqueles que tiveram glicemia de jejum e TOTG alterados eram mais velhos e tinham mais condições de risco para DM em relação aos que estavam dentro dos limites da normalidade; o estudo também demonstrou a falta de concordância entre os dois testes utilizados, sendo que entre aqueles com intolerância à glicose, $18 \%$ apresentavam glicemia de jejum dentro dos valores normais ${ }^{18}$.

É importante verificar as possíveis diferenças nas prevalências segundo raça/cor, pois os níveis de HbAlc reconhecidamente são variados em diferentes populações ${ }^{2}$, especialmente nos afrodescendentes ${ }^{19}$. A população negra, neste estudo, apresentou prevalências superiores de pré-diabetes/hiperglicemia intermediária, o que poderia estar relacionado a questões genéticas, características fisiológicas dos glóbulos vermelhos e turnover celular ${ }^{20}$. Estudos com diferentes populações têm verificado maiores níveis de HbAlc na população de cor preta quando comparada à branca, mesmo após controle para outros fatores como idade, acesso aos serviços de saúde e nível socioeconômico ${ }^{20}$. Estudo com população negra 
da África do Sul indicou que, para esta população, o ponto de corte ideal para deteç̧ão de diabetes pela $\mathrm{HbAlc}$ seria $6,0 \%$ ( $42 \mathrm{mmol} / \mathrm{mol}$ ), independentemente do teste referência ser o TOTG ou a glicose de jejum ${ }^{21}$. Esse resultado sugere que a detecção de pré-diabetes/hiperglicemia intermediária com os valores adotados nesse estudo, já poderiam ser indicativos de diabetes nessa população.

Cabe ressaltar que a população brasileira é bastante miscigenada, sendo que resultados nacionais podem não ser comparáveis àqueles realizados fora do país, onde outras etnias predominam. Além disso, a variável raça/cor, seguindo o padrão do Instituto Brasileiro de Geografia e Estatísticas (IBGE), foi autodeclarada. Uma avaliação com índios xavantes da região Centro-Oeste, considerados de alto risco para diabetes, verificou um desempenho moderado (acurácia de 51,4\%) da HbA1c para detecção de tolerância à glicose diminuída nesta população, pelo intervalo de $5,7-6,4 \%$.

Estudos anteriores demonstraram maior prevalência de diabetes nas regiões Sudeste e Centro-Oeste ${ }^{10}$. Quando considerada a informação autorreferida, no entanto, as Regiões Sul e Sudeste do Brasil em geral apresentam prevalências maiores; este fato poderia estar relacionado ao melhor acesso ao diagnóstico e aos cuidados em saúde desta população ${ }^{23}$. Neste estudo, após ajuste das estimativas por diversos fatores como idade, escolaridade, raça e IMC da população, a prevalência de pré-diabetes, pelo critério da ADA, e de hiperglicemia intermediária, considerando critério da OMS, foi maior na região Sudeste do país, sugerindo que outros fatores, não identificados nesta análise, estejam relacionados à hiperglicemia na população daquela região.

$\mathrm{O}$ uso da HbAlc para diagnóstico ainda é controverso. Uma revisão sistemática indicou uma sensibilidade de $49 \%$ e especificidade de $79 \%$, com acurácia geral estimada em $71 \%$, no entanto o desempenho do teste mostrou-se heterogêneo de acordo com a população e o local do estudo ${ }^{15}$. Por outro lado, o argumento para a inclusão da HbA1c como método de rastreamento e diagnóstico está na possibilidade de verificar uma exposição de longo prazo (de dois a três meses) a níveis glicêmicos elevados (basal e pós -prandial), podendo refletir uma combinação de condições fisiopatológicas subjacentes à glicemia de jejum alterada e tolerância à glicose diminuí$\mathrm{da}^{1,2}$. Considerando ainda a logística e os custos da realização de medidas bioquímicas em nível populacional, o uso da HbAlc torna-se conve- niente para fins epidemiológicos, por dispensar o jejum ${ }^{10}$, indicando-se medidas individualizadas para uso na clínica, incluindo-se a realização de testes confirmatórios previamente à instituição de tratamento específico.

O rastreamento de estados intermediários de hiperglicemia e pré-diabetes também é controverso, especialmente porque a efetividade do rastreamento seguido de programa intensivo para mudança de estilo de vida foi verificada apenas quando a glicemia intermediária foi testada por um TOTG, e tal rastreamento é pouco utilizado para lograr alcance populacional. Comparação recente em brasileiros mostrou que os critérios da OMS para hiperglicemia intermediária seriam mais preditivos de diabetes ${ }^{24}$.

Como limitações deste estudo deve-se considerar que a falta de consenso para determinação de pré-diabetes e hiperglicemia intermediária entre as maiores entidades mundiais impede a determinação de uma estimativa única nacional. Ainda, a transversalidade dos dados do inquérito permite apenas a análise de associações e não de causalidade. Pela característica do estudo, a impossibilidade de realização de testes considerados de referência, não permitiu uma análise detalhada dos critérios diagnósticos e da acurácia da hemoglobina glicada. Cabe ressaltar também que, conforme indicado pela literatura ${ }^{20}$, valores de HbAlc podem sofrer influência de hemoglobinopatias, anemias e outras condições, não abordadas neste estudo. O percentual de perdas na realização das amostras laboratoriais, principalmente em virtude de endereços não encontrados, reduziu a população real estudada. No entanto, pesos de pós-estratificação foram aplicados de forma a manter a representatividade amostral.

\section{Conclusões}

Este estudo aponta um intervalo entre 7,5 a $18,5 \%$ de adultos brasileiros que apresentam pré-diabetes / hiperglicemia intermediária, além de identificar um escore de risco para a ocorrência dessa condição. Considerando as limitações e os fatores influentes nas medidas de hemoglobina glicada, a identificação da presença de um conjunto de fatores de risco comuns à ocorrência de diabetes e suas complicações, tais como idade, etnia e marcadores biológicos, como excesso de peso e circunferência abdominal aumentada, hipertensão arterial e baixo colesterol HDL - pode auxiliar na recomendação de exames e análises específicos em cada estrato populacional, de for- 
ma a melhor definir grupos prioritários para intervenções oportunas.

Dessa forma, o estudo cumpre seu propósito epidemiológico de atualizar as estimativas nacionais sobre uma parcela importante da população considerada de alto risco para o desenvolvimento de diabetes, subsidiando as ações de planejamento e vigilância em saúde, alcançando também um objetivo da própria PNS.

\section{Colaboradores}

BPM Iser participou da concepção e delineamento do estudo, organização, análise e interpretação dos dados e foi responsável pela primeira versão do manuscrito. PC Pinheiro foi responsável pela extração e análise de dados, contribuiu para a interpretação dos mesmos, e para a escrita do manuscrito. DC Malta, BB Duncan e MI Schmidt contribuíram para a concepção e delineamento do estudo, análise e interpretação dos dados, e participaram da escrita científica. Todos os autores aprovaram a versão final a ser publicada, sendo responsáveis por seu conteúdo.

\section{Referências}

1. American Diabetes Association. Classification and Diagnosis of Diabetes: Standards of Medical Care in Diabetes-2020. Diabetes Care 2020; 43(Supl. 1):S1431.

2. Sequeira IR, Poppitt SD. HbAlc as a marker of prediabetes: A reliable screening tool or not. Insights in Nutrition and Metabolism [Internet]. 2017 [acessado 2020 Maio 12]; 1(1). Disponível em: https://www.alliedacademies.org/abstract/hbalc-as-a-marker-of-prediabetes-a-reliable-screening-tool-or-not-7025.html

3. Zhang X, Gregg EW, Williamson DF, Barker LE, Thomas W, Bullard KM, Imperatore G, Williams DE, Albright AL. AlC level and future risk of diabetes: a systematic review. Diabetes Care 2010; 33(7):1665-1673.

4. World Health Organization (WHO). Definition and diagnosis of diabetes mellitus and intermediate hyperglycaemia [Internet]. WHO; 2006 [acessado 2020 Maio 13]. 46 p. Disponível em: https://www.who.int/ diabetes/publications/diagnosis_diabetes2006/en/

5. Hostalek U. Global epidemiology of prediabetes - present and future perspectives. Clin Diabetes Endocrinol [Internet] 2019 [acessado 2020 Maio 13]; 5. Disponível em: https://www.ncbi.nlm.nih.gov/pmc/articles/ PMC6507173/

6. Saeedi P, Petersohn I, Salpea P, Malanda B, Karuranga S, Unwin N, Colagiuri S, Guariguata L, Motala AA, Ogurtsova K, Shaw JE, Bright D, Williams R, On behalf of the IDF Diabetes Atlas Committee. Global and regional diabetes prevalence estimates for 2019 and projections for 2030 and 2045: Results from the International Diabetes Federation Diabetes Atlas, $9^{\text {th }}$ edition. Diabetes Research and Clinical Practice [Internet]. 2019 [acessado 2020 Maio 12]; 157. Disponível em: https:// www.diabetesresearchclinicalpractice.com/article/ S0168-8227(19)31230-6/abstract

7. Sociedade Brasileira de Diabetes. Diretrizes da Sociedade Brasileira de Diabetes 2019-2020 [Internet]. SBD; 2019 [acessado 2020 Maio 12] p. 490. Disponível em: https://www.diabetes.org.br/profissionais/images/DIRETRIZES-COMPLETA-2019-2020.pdf 
8. World Health Organization (WHO). Use of glycated haemoglobin (HbAlc) in the diagnosis of diabetes mellitus. Diabetes Res Clin Pract 2011; 93(3):299-309.

9. Szwarcwald CL, Malta DC, Pereira CA, Vieira MLFP, Conde WL, Souza Júnior PR, Damacena GN, Azevedo LO, Azevedo E Silva G, Theme Filha MM, Lopes CS, Romero DE, Almeida WS, Monteiro CA. National Health Survey in Brazil: design and methodology of application. Cien Saude Colet 2014; 19(2):333-342.

10. Malta DC, Duncan BB, Schmidt MI, Machado IE, Silva AG, Bernal RTI, Pereira CA, Damacena GN, Stopa SR, Rosenfeld LG, Szwarcwald CL. Prevalência de diabetes mellitus determinada pela hemoglobina glicada na população adulta brasileira, Pesquisa Nacional de Saúde. Rev Bras Epidemiol [Internet]. 2019 [acessado 2020 Maio 29]; 22. Disponível em: http://www. scielo.br/scielo.php?script=sci_abstract\&pid=S1415 $-790 X 2019000300408 \& \operatorname{lng}=\mathrm{en} \& \mathrm{nrm}=\mathrm{iso} \& \mathrm{t} \operatorname{lng}=\mathrm{pt}$

11. Iser BPM, Stopa SR, Chueiri PS, Szwarcwald CL, Malta DC, Monteiro HOC, Duncan, Bartholow BD, Schmidt MI. Prevalência de diabetes autorreferido no Brasil: resultados da Pesquisa Nacional de Saúde 2013. Epidemiologia e Serviços de Saúde 2015; 24(2):305314.

12. Iser BPM, Vigo A, Duncan BB, Schmidt MI. Trends in the prevalence of self-reported diabetes in Brazilian capital cities and the Federal District, 2006-2014. Diabetology and Metabolic Syndrome [Internet]. 2016 [acessado 2020 Maio 29]; 8(1). Disponível em: http:// www.embase.com/search/results?subaction=viewrecord\&from $=$ export\&id $=\mathrm{L} 612834024$

13. Stopa SR, Cesar CLG, Segri NJ, Alves MCGP, Barros MBA, Goldbaum M. [Prevalence of arterial hypertension, diabetes mellitus, and adherence to behavioral measures in the city of São Paulo, Brazil, 2003-2015]. Cad Saude Publica 2018; 34(10):e00198717.

14. Miraglia JL, Mafra ACCN, Monteiro CN, Borges LM. The variation of the burden of hypertension and diabetes in two large districts of the city of São Paulo, Brazil, based on primary health care routinely-collected data. PLoS One 2019; 14(3):e0213998.

15. Barry E, Roberts S, Oke J, Vijayaraghavan S, Normansell R, Greenhalgh T. Efficacy and effectiveness of screen and treat policies in prevention of type 2 diabetes: systematic review and meta-analysis of screening tests and interventions. BMJ 2017; 356:16538.

16. Malerbi DA, Franco LJ. Multicenter study of the prevalence of diabetes mellitus and impaired glucose tolerance in the urban Brazilian population aged 30-69 yr. The Brazilian Cooperative Group on the Study of Diabetes Prevalence. Diabetes Care 1992; 15(11):1509-1516.

17. Moreira NCV, Montenegro RM, Meyer HE, Bhowmik B, Mdala I, Siddiquee T, Fernandes VO, Hussain A. Glycated Hemoglobin in the Diagnosis of Diabetes Mellitus in a Semi-Urban Brazilian Population. Int $J$ Environ Res Public Health 2019; 16(19):3598.
18. Matos LN, Giorelli GV, Saado A, Dias CB. Prevalence of prediabetes in patients with metabolic risk. Sao Paulo Med J 2011; 129(5):300-308.

19. Ziemer DC, Kolm P, Weintraub WS, Vaccarino V, Rhee MK, Twombly JG, Narayan KMV, Koch DD, Phillips LS. Glucose-independent, black-white differences in hemoglobin Alc levels: a cross-sectional analysis of 2 studies. Ann Intern Med 2010; 152(12):770-777.

20. Herman WH, Cohen RM. Racial and ethnic differences in the relationship between $\mathrm{HbAlc}$ and blood glucose: implications for the diagnosis of diabetes. J Clin Endocrinol Metab 2012; 97(4):1067-1072.

21. Hird TR, Pirie FJ, Esterhuizen TM, O'Leary B, McCarthy MI, Young EH, Sandhu MS, Motala AA. Burden of Diabetes and First Evidence for the Utility of HbAlc for Diagnosis and Detection of Diabetes in Urban Black South Africans: The Durban Diabetes Study. PLoS One 2016; 11(8):e0161966.

22. Franco LJ, Dal Fabbro AL, Martinez EZ, Sartorelli DS, Silva AS, Soares LP, Franco LF, Kuhn PC, Vieira-Filho JPB, Moisés RS. Performance of glycated haemoglobin $(\mathrm{HbAlc})$ as a screening test for diabetes and impaired glucose tolerance (IGT) in a high risk population--the Brazilian Xavante Indians. Diabetes Res Clin Pract 2014; 106(2):337-342.

23. Malta DC, Stopa SR, Iser BPM, Chueiri PS, Duncan BB, Schmidt MI, Duncan BB. Cuidados em saúde entre portadores de diabetes mellitus autorreferido no Brasil, Pesquisa Nacional de Saúde, 2013. Rev Bras Epidemiol 2015; 18(Supl. 2):17-32.

24. Schmidt MI, Bracco PA, Yudkin JS, Bensenor IM, Griep RH, Barreto SM, Castilhos CD, Duncan BB. Intermediate hyperglycaemia to predict progression to type 2 diabetes (ELSA-Brasil): an occupational cohort study in Brazil. Lancet Diabetes Endocrinol 2019; 7(4):267-277.

Artigo apresentado em 14/09/2020

Aprovado em 14/09/2020

Versão final apresentada em 16/09/2020

Editores chefes: Editores-chefes: Maria Cecília de Souza Minayo, Romeu Gomes, Antônio Augusto Moura da Silva 